\title{
Retreatment of Hepatitis C Patients Who Previously Experienced Treatment Failure
}

\author{
Fernando Lopes Gonçales Jr. \\ Department of Viral Hepatitis Studies, State University of Campinas (Unicamp); Campinas, SP, Brazil
}

In its different formulations, interferon (IFN) alpha combined with ribavirin (RBV) is the best treatment alternative for patients infected with the hepatitis $\mathrm{C}$ virus (HCV) [1]. In such patients, the objective is to achieve an end-of-treatment virological response - negative serum HCV ribonucleic acid (RNA) - followed by a sustained virological response (SVR), which is defined as HCV RNA negativity for six months after the suspension of treatment.

The treatment regimens for HCV infection have evolved from monotherapy with conventional IFN alpha to combined therapy with IFN and RBV and, more recently, to combined therapy with pegylated IFN (PEG-IFN alpha-2a or alpha-2b) and RBV $[2,3]$. It is known that approximately $60 \%$ of the patients infected with HCV genotype 1 and nearly 40\% of those infected with genotype 3 do not achieve an SVR when treated with the conventional regimen of IFN and RBV [2-7]. The availability of PEG-IFNs has reduced the percentage of patients experiencing treatment failure. In large international trials involving treatment-naïve patients, regimens that include the combination of PEG-IFN and RBV have produced significantly higher rates of SVR than those observed with the use of the conventional combination of IFN and RBV (5456\% vs. 44-47\%) [5-7].

Similar to what occurs in treatment-naïve patients, retreatment provides the patient with a new chance to achieve an SVR. Long-term studies have proven that the vast majority of the patients who achieve an SVR usually remain negative for HCV RNA for a long time [8].

The arguments in favor of retreating patients would be the possibility of eradicating HCV, reducing fibrosis, and decreasing the risk of evolving to hepatocarcinoma. With regard to the new antiviral agents, we do not know when they will be available or whether all patients will be able to wait several years to initiate retreatment.

It is known that patients who experience relapse after treatment with conventional IFN, with or without RBV, respond better to retreatment with PEG-IFN alpha and RBV than do those presenting no response to treatment with conventional IFN (with or without RBV). Krawitt et al. observed an SVR rate of 55\% in 66 relapsing patients who were retreated with PEGIFN alpha-2b (100-150 $\mu \mathrm{g} /$ week) and RBV (1000 mg/day), compared with only $20 \%$ in 116 previous nonresponders treated with the same regimen [9]. An SVR was observed in $53 \%$ of the relapsing patients infected with genotype 1 , compared with $59 \%$ of the relapsing patients infected with genotypes 2 or 3 . There were, therefore, no significant differences between these groups of patients. This was not

The Brazilian Journal of Infectious Diseases 2007;11 (5) Suppl. 1:50-51. (C) 2007 by The Brazilian Journal of Infectious Diseases and Contexto Publishing. All rights reserved. observed in the previous nonresponders receiving retreatment. Of those, only $17 \%$ of the individuals infected with genotype 1 , in comparison with $57 \%$ of the individuals infected with genotypes 2 or 3, achieved an SVR. Therefore, genotype had an influence on SVR in the previous nonresponders who underwent retreatment.

Studies conducted in Brazil and involving nonresponders to IFN/RBV demonstrated SVR rates resulting from retreatment with the PEG-IFN alpha-2a + RBV regimen [10], as well as with the PEG-IFN alpha-2b + RBV regimen [11], that were higher than those reported in international studies. Of the relapsers treated, 57\% [10] and 62\% [11] achieved an SVR. These SVR rates in relapsers are higher than, for example, the rates of 41\% and 59\% obtained, respectively, in Canada (by Sherman et al.) and in France (by Moucari et al.) [12,13]. In analyzing the response in relapsers by genotype, the Brazilian researchers found that an SVR was achieved in $69-70 \%$ of those infected with genotype 3, compared with $43-44 \%$ of those infected with genotype 1 [10,11].

True nonresponders, that is, patients who never presented viral negativity during or at the end of the treatment, are the most difficult group to retreat. Some authors showed that, after previous treatment with conventional IFN, with or without RBV, relapsers have significantly higher SVR when retreated with PEG-IFN alpha-2b and RBV than do nonresponders to previous treatment (55\% vs. 20\%; $\mathrm{p}<0.001$ ) [9]. The same was observed by Sherman et al., who found that, after retreatment with PEG-IFN alpha-2a and RBV, the SVR rate was 23\% in nonresponders and $41 \%$ in relapsers [12]. The results of the retreatment of true nonresponders to PEG-IFN and RBV obtained by the Brazilian researchers are better than those reported in international studies [10,11]. However, it is very difficult to compare different studies with different populations of patients treated with different formulations of conventional IFN.

Two large international trials (EPIC3 and HALT-C) are evaluating, in two different international cohorts, the responses to treatment with the combination of PEG-IFN and $\mathrm{RBV}$ in nonresponders to previous treatment with IFN and RBV [14,15]. The EPIC3 study is also evaluating the real meaning of the virological response at week 12 and its predictive value in nonresponders. The final results of both trials will soon be available.

With regard to the use of induction doses of PEG-IFN in the first 12 weeks or the increase in the duration of the treatment of nonresponders, there are few studies in the literature.

In a recent pilot study, researchers working in Spain observed that patients with genotype $1 \mathrm{HCV}$ who were retreated for 72 weeks, with different induction doses of PEG- 
IFN alpha-2a in the first 12 weeks, achieved an SVR at a rate of $30-37 \%$, which is practically twice as high as the SVR rate of $18 \%$ observed in those treated with normal doses of PEGIFN alpha-2a [16].

Since nonresponders to initial treatment/relapsers constitute a very heterogeneous group, it is necessary to carefully qualify and select the patients who should be retreated. The various factors that might have been responsible for the lack of a response should be modified/ nullified prior to the initiation of or even during the new treatment cycle. Currently, the patients who are considered the least likely to respond to retreatment are the true nonresponders, as well as those who are black, infected with genotype 1 , presenting a high viral load, having advanced liver disease, or presenting intercurrent diseases, such as obesity, HIV coinfection, and coinfection with hepatitis B virus.

Those who were previously treated with IFN as monotherapy or with the combination of IFN and RBV are more likely to achieve an SVR than are the nonresponders to the combination of PEG-INF and RBV. Those who had relapses during (breakthrough) or after the previous treatment also fare better than do those who are true nonresponders. Patients who were noncompliant with the previous treatment or those who needed to undergo IFN or RBV dose reduction due to cytopenias or other adverse effects usually respond better to retreatment than do those who received full doses. It is clear that the factors responsible for noncompliance should be corrected. In addition, medication dose reductions should be adequately addressed. In nonresponders, erythropoietin and filgrastim should be used earlier and further dose reductions should be avoided at all costs. Drug and alcohol users who, due to their dependence, did not satisfactorily complete all phases of the previous treatment might respond better to retreatment if these cofactors are removed. This also applies to the patients who did not have adequate social or cultural support. Obese patients, insulin-resistant patients, dyslipidemic patients, patients with steatosis, and patients with liver diseases, such as hemochromatosis, should be retreated, preferably after the diagnosis and treatment of these accompanying conditions have been appropriately addressed. The doses of PEG-IFN should be the same as those used in treatment-naïve patients. We recommend that, in retreatment, the doses of RBV be as high as possible. Due to the paucity of studies of large population samples, the duration of retreatment should be 48 weeks for all genotypes. At the moment, there are no conclusive data in the literature to support the use of higher doses of PEGIFN and RBV, the use of induction doses, or the extension of treatment duration to more than 48 weeks in such patients. With regard to the week-12 rule, there is strong evidence that patients who do not present HCV RNA negativity by that time are much less likely to achieve an SVR, and that their treatment should therefore be discontinued.

\section{References}

1. National Institutes of Health Consensus Development Conference Statement: Management of hepatitis C: 2002-June 10-12, 2002. Hepatology 2002;36:S3-20.

2. McHutchison J.G., Gordon S.C., Schiff E.R., et al. Interferon alfa-2b alone or in combination with ribavirin as initial treatment for chronic hepatitis C. $N$ Engl J Med 1998;339:1485-92.

3. Poynard T., Marcellin P., Lee S.S., et al. Randomised trial of interferon alpha-2b plus ribavirin for 48 weeks or for 24 weeks versus interferon alpha-2b plus placebo for 48 weeks for treatment of chronic infection with hepatitis $C$ virus. Lancet 1998;352:1426-32.

4. Poynard T., McHutchison J., Goodman Z., et al. Is an “a la carte” combination interferon alfa-2b plus ribavirin regimen possible for the first line treatment in patients with chronic hepatitis C? Hepatology 2000;31:211-8.

5. Fried M.W., Shiffman M.L., Reddy K.R., et al. Peginterferon alfa-2a plus ribavirin for chronic hepatitis $\mathrm{C}$ virus infection. N Engl J Med. 2002;347:975-82.

6. Manns M.P., McHutchison J.G., Gordon S.C., et al. Peginterferon alfa-2b plus ribavirin compared with interferon alfa-2b plus ribavirin for initial treatment of chronic hepatitis C: a randomised trial. Lancet 2001;358:958-65.

7. Hadziyannis S.J., Sette H., Morgan T.R., et al. Peginterferonalpha2a and ribavirin combination therapy in chronic hepatitis C: a randomized study of treatment duration and ribavirin dose. Ann Intern Med 2004;140:346-55.

8. Marcellin P., Boyer N., Gervais A., et al. Long-term histologic improvement and loss of detectable intrahepatic HCVRNA in patients with chronic hepatitis C and sustained response to interferon-alpha therapy. Ann Int Med 1997; $127: 875-81$.

9. Krawitt E.L., Ashikaga T., Gordon S.R., et al. Peginterferon alpha-2b and ribavirin for treatment-refractory chronic hepatitis C. J Hepatol 2005;43:243-9.

10. Parise E., Cheinquer H., Crespo D., et al. Peginterferon alfa-2 a (40 KD) (PEGASYS) plus ribavirin (COPEGUS) in retreatment of chronic hepatitis $\mathrm{C}$ patients, nonresponders and relapsers to previous conventional interferon plus ribavirin therapy. Braz J Infect Dis 2006;10:11-6.

11. Gonçales F.L. Jr., Vigani A., Gonçales N., et al. Weight-based combination therapy with peginterferon alpha-2b and ribavirin for naïve, relapser and non-responder patients with chronic hepatitis C. Braz J Infect Dis 2006;10:311-6.

12. Sherman M., Yoshida E.M., Deschenes M., et al. Peginterferon alfa-2a (40KD) plus ribavirin in chronic hepatitis $C$ patients who failed previous interferon therapy. Gut 2006;55:1631-8.

13. Moucari R., Ripault M.P., Oules V., et al. High predictive value of early viral kinectics in retreatment with peginterferon and ribavirin of chronic hepatitis $\mathrm{C}$ patients non-responders to standard combination therapy. J Hepatol 2007;46:596-604.

14. Shiffman M.L., Di Bisceglie A.M., Lindsay K.L., et al. Peginterferon alfa-2a and ribavirin in patients with chronic hepatitis $C$ who have failed prior therapy. Gastroenterology 2004; 126 : $1015-23$.

15. Poynard T., Schiff E., Terg R., et al. Sustained virologic response (SVR) in the EPIC3 trial: week 12 virology predicts $\mathrm{SVR}$ in previous interferon/ribavirin treatment failures receiving Peg-Intron/Rebetol weight based dosing. J Hepatol 2005;42 (Suppl. 2):40.

16. Diago M. Peginterferon alfa-2a (40kd) (Pegasys $\left.{ }^{\circledR}\right)$ and ribavirin (Copegus ${ }^{\circledR}$ ) in patients infected with $\mathrm{HCV}$ genotype 1 who failed to respond to interferon and ribavirin: final results of the Spanish high-dose induction pilot trial. Presented at the $55^{\text {th }}$ AASLD; October 29-November 2, 2004; Boston, MA. 